\title{
Simulated Motion Enhances Neuronal Selectivity for a Sound Localization Cue in Background Noise
}

\author{
Terry T. Takahashi and Clifford H. Keller \\ Institute of Neuroscience, University of Oregon, Eugene, Oregon 97403
}

In nature, sound sources move and signals are accompanied by background noise. Noting that motion helps the perception of visual stimuli, we tested whether motion similarly facilitates the detection of acoustic targets, at the neuronal level. Auditory neurons in the central nucleus of the barn owl's inferior colliculus (ICc), due to their selectivity for interaural phase difference $(\Delta \phi)$, are sharply tuned to the azimuth of sound sources and are arrayed to form a topographic map of $\Delta \phi$. While recording from single ICc neurons, we presented tones that simulated either moving or stationary sound sources with and without background noise.

We found that the tuning of cells in the ICc for $\Delta \phi$ was sharper for stimuli that simulated motion than for those that simulated stationary targets. The neurons signaled the presence of a tone obscured by noise better if the tone moved than if the tone remained stationary. The resistance to noise observed with moving stimuli could not be reproduced with the temporal modulation of the stimulus amplitude, suggesting that a change of position over time was required.

Motion plays an important role in visual perception. As early as 1878 , it was demonstrated that shadows that were too dim to detect when stationary became visible when moved, suggesting that motion aids the detection of visual stimuli (Schneider, 1878, as cited in Boring, 1948). More recently, studies have shown that movement or flicker of a pattern is detectable at contrasts that are too low for identification of the pattern (Kulikowski and Tolhurst, 1973). Other studies have demonstrated that motion is critical for the extraction of figure from ground, the most notable demonstration being the random-dot kinematogram in which the coherent motion of a subset of randomly positioned dots causes the outlines of an object to stand out from the background (Anstis, 1970; Braddick, 1974).

Does the motion of sound sources similarly facilitate their detection? In man, who is relatively insensitive to acoustic motion (Grantham, 1986), the motion of a sound source does not facilitate its detection (Wilcott and Gales, 1954; Robinson, 1971; Grantham and Luethke, 1988). By contrast, certain echolocating bats are better able to avoid obstacles and detect targets amidst

\footnotetext{
Received Mar. 3, 1992; revised May 21, 1992; accepted June 8, 1992.

We thank Jim Gent and Jack Vanderlip for veterinary care; Julie Prather, Yvonne Hall, and Monte Matthews for owl husbandry; John Martin for help with computer software; and Mike P. Fleming for technical help. Petr Janata, Shig Kuwada, Teri Iawton, Rich Marrocco, and Malcolm Semple provided many helpful discussions and criticisms. This study was supported by grants from the National Institutes of Health and the Office of Naval Research.

Correspondence should be addressed to Terry $\mathbf{T}$. Takahashi and Clifford $\mathbf{H}$ Keller at the above address.

Copyright (C) 1992 Society for Neuroscience $0270-6474 / 92 / 124381-10 \$ 05.00 / 0$
}

clutter if the objects move (Simmons, 1981; McCarty and Jen, 1983).

The barn owl (Tyto alba), although lacking the bat's ability to echolocate, is nevertheless a keen auditory predator capable of striking moving prey in absolute darkness (Payne, 1971). Its brain has considerable specializations for such purposes. Most remarkably, auditory neurons in its inferior colliculus have discrete spatial receptive fields and are systematically arrayed to form a topographic map of space (Knudsen and Konishi, 1978; Wagner et al., 1987). Some of these neurons, moreover, are sensitive to the direction of stimulus motion along the horizon (Wagner and Takahashi, 1990). Thus, despite the negative findings in man, we expected the owl's auditory system to exhibit adaptations for the exploitation of acoustic motion.

In the present study, we recorded from single auditory neurons in the central nucleus of the barn owl's inferior colliculus (ICc) that are responsive to tonal stimuli and are selective for interaural phase difference $(\Delta \phi)$, the binaural cue correlated with stimulus azimuth (Knudsen and Konishi, 1979; Moiseff and Konishi, 1981a). Within the ICc, these neurons form a topographic map of the horizontal meridian (Wagner et al., 1987). The responses of neurons in the ICc to stimuli that simulated tones from moving or stationary targets were compared under noisy and quiet background conditions.

We simulated a moving source by presenting tones of slightly differing frequencies to each ear. Under these conditions, $\Delta \phi$ changes smoothly over time, as would a tone from a source moving in an arc along the horizon, at a constant angular speed. In man, this stimulus paradigm is known to produce an illusion of motion, and the perception is termed "binaural beats." The analogous psychophysical studies have not been done in owls. However, since the stimulus configuration generates smooth changes in $\Delta \phi$ and owls use $\Delta \phi$ as the cue for the horizontal location of the source, we will use the terms binaural beats, moving tones, and motion interchangeably.

\section{Materials and Methods}

Surgery and animal care. All procedures were carried out under a protocol approved by the Institutional Animal Care and Use Committee of the University of Oregon. Adult barn owls (Tyto alba; $N=6$ ) were anesthetized by intramuscular injections of ketaminc $(0.1 \mathrm{ml} / \mathrm{hr}$; Vctalar, $100 \mathrm{mg} / \mathrm{ml}$; Parke-Davis) and diazepam $(0.05 \mathrm{ml} / \mathrm{hr}$; Diazepam C-IV, $5 \mathrm{mg} / \mathrm{ml}$; LyphoMed) and given a prophylactic dosage of ampicillin $(0.2 \mathrm{ml}$, i.m.; Polyflex, $250 \mathrm{mg} / \mathrm{ml}$; Aveco). The owl was placed into a stereotaxic device that held its head tilted downward at a $45^{\circ}$ angle. The scalp was infused with a local anesthetic $(2 \%$ lidocaine $\mathrm{HCl}$; Xylocaine, Astra Pharmaceuticals) and incised, and a stainless steel plate was cemented to the rostral portion of the cranium. After the plate was firmly attached, the ear bars that held the head in position were replaced with miniature earphones. A hole about $0.7 \mathrm{~cm}^{2}$ was opened in the skull with sterile rongeurs. Heavy mineral oil (Spectrum Chemical) was ap- 
plied to the dural surface to prevent desiccation. Body temperature was monitored and was maintained with a circulating-water heating pad. Fluid levels were maintained by periodic subcutaneous injections of 5\% dextrose in lactated Ringer's solution (Abbot Labs).

As we were attempting to document a new phenomenon, we surveyed the ICc of each bird extensively. In order to minimize the stress on the bird, we ran two 15-20 hr sessions separated by a 4-7 d recovery period, instead of a single lengthy procedure. At the end of the first session, the craniotomy was closed with dental cement (Vitrabond, 3M Inc.) and the scalp was sutured and covered with a topical antibacterial cream (Bacitracin-Neomycin-Polymyxin Ointment, E. Fougerra and Co.). Before returning the bird to a recovery cage, it was given $0.2 \mathrm{cc}$ of dexamethasone and $0.2 \mathrm{cc}$ of vitamin B complex intramuscularly and 10$20 \mathrm{cc}$ of $5 \%$ dextrose in lactated Ringer's solution intravenously. The recovery cage was maintained at $34^{\circ} \mathrm{C}, 25 \%$ humidity, and $30 \% \mathrm{O}_{2}$. The bird's activity was monitored hourly until it fully recovered.

Birds were killed at the end of the second session by an overdose of pentobarbital (Nembutal, Abbot Laboratories) and were exsanguinated $(0.9 \% \mathrm{NaCl})$ and fixed $(3.75 \%$ paraformaldehyde in $0.1 \mathrm{M}$ phosphate buffer, $\mathrm{pH}$ 7.4) transcardially. The brains were removed and processed for histology to confirm the locations of the electrode penetrations.

Electrode locations relative to the cytoarchitectural boundaries of the inferior colliculus were confirmed by histological examination to be within the ICc (for descriptions of the inferior colliculus and surrounding structures, see Takahashi et al., 1987; Takahashi and Konishi, 1988a,b).

Stimulus generation and data analysis. Tonal stimuli were produced by a dual-channel synthesizer (Hewlett Packard 3245A Universal Source). The analog signals were scaled down with programmable attenuators (Modular Instruments Inc.) to achieve average binaural levels that were 20-30 dB above neuronal threshold. The attenuated signals were amplified with a stereophonic amplifier (Mcintosh 754) and transduced with miniature earphones matched in amplitude and phase (Sony MDRE272).

In order to produce tones with continuously varying $\Delta \phi$, sine waves with slightly differing frequencies were presented to the left and right ears (Kuwada et al., 1979; Moiseff and Konishi, 1981a; Yin and Kuwada, 1983; Moiseff and Haresign, 1992). The sounds start out inphase binaurally, but over time the phase angle in the ear receiving the higher frequency advances relative to that in the opposite ear, until they are once again in phase. This simulates a source moving in an arc toward the higher-frequency ear with an angular speed proportional to the absolute value of the interaural frequency difference $(\Delta F)$ and the period of the stimulus tone $(\tau)$, which, in our tests, was at a cell's best frequency:

$$
\text { Angular speed }=|\Delta F| \tau / k \text {. }
$$

The term $k$ is a constant relating interaural time difference (ITD) to azimuth and has been computed to be $2.3 \mu \mathrm{sec}$ of ITD per degree of azimuth for the adult barn owl (Moiseff, 1989). A graphic representation of binaural beats is shown in a report by Kuwada et al. (1979; see also Yin and Kuwada, 1983).

All cells of our sample ( $N=59$ in 6 birds) were tested with stimuli simulating rightward and leftward motion. All but 10 of the cells were tested with $\Delta F$ values of 1,2 , or $4 \mathrm{~Hz}$. Using Equation 1, the angular speeds simulated for these neurons ranged from $52^{\circ}$ of azimuth per second $(\Delta F=1 \mathrm{~Hz} ; \tau=120 \mu \mathrm{sec})$ to $278^{\circ}$ of azimuth per second $(\Delta F$ $=4 \mathrm{~Hz} ; \tau=160 \mu \mathrm{sec})$. This is within the range of angular speeds encountered by the owl during flight or the movement of prey. Of the 10 remaining cells, one was tested at $6 \mathrm{~Hz}$, eight at $8 \mathrm{~Hz}$, and one at $16 \mathrm{~Hz}$, resulting in angular speeds of up to $1360^{\circ}$ of azimuth per second $(\Delta F=8 \mathrm{~Hz} ; \tau=391 \mu \mathrm{sec})$.

Moving tones lasted for $11.5 \mathrm{sec}$, during which time we recorded spikes. Spikes occurring during the first 1.5 beat cycles and after the offset of the stimulus were discarded in order to avoid neuronal responses to stimulus onset and offset. The spike times were binned with respect to the period of the binaural beat so as to plot the number of spikes as a function of $\Delta \phi$ at a resolution of $1 / 41$ th of a cycle. The vector strength, a measure of tuning to $\Delta \phi$, was computed from these histograms (Goldberg and Brown, 1969; Kuwada and Yin, 1983). A vector strength of 1 indicates that all spikes are in one bin, and a vector strength of $U$ indicates that all spikes are evenly distributed throughout all bins. We interpreted the vector strength as the degree to which a cell can detect and signal the presence of a stimulus.

Stationary signals consisted of $1 \mathrm{sec}$ tone bursts of identical frequencies presented to the left and right ears. The $\Delta \phi$ remained fixed for the duration of a burst and was incremented between bursts in 41 steps from +0.5 to -0.5 cycles. The number of spikes for each tone burst was plotted as a function of $\Delta \phi$, and the resulting histogram was analyzed as described above for binaural beats. Spikes occurring during the first $20 \mathrm{msec}$ and after the end of each tone burst were discarded to avoid onset and offset responses.

Stationary tonal stimuli were also amplitude modulated for some tests. In these cases, the tones at each $\Delta \phi$ were multiplied by a triangular envelope from a digital-to-analog converter (Modular Instruments Inc.). The envelopes were binaurally inphase, had frequencies equal to the $\Delta F$ used in that cell's binaural beats test, and modulated the tones to a depth of $75 \%$. Histograms of spike counts versus $\Delta \phi$ for these amplitude modulated (AM) tones were then compiled and analyzed as discussed above.

Signals were embedded in noise by summing the analog output of the signal generators of the left and right sides with broad-band noise having a flat spectrum (after transduction) between 1 and $10 \mathrm{kHz}$. The noises to the ears were taken from two, independent, random-noise generators and were therefore binaurally uncorrelated. The signal-to-noise ratio $(S / N)$ was computed as the quotient of the root-mean-square energy in the signal band to that in the noise from 1 to $10 \mathrm{kHz}$. The total energy was kept constant for the different $S / N$ values tested.

The responses of our neurons under various test conditions were compared using the Wilcoxon paired signed-ranks test (Sokal and Rohlf, 1981) except where noted otherwise.

\section{Results}

\section{General properties of ICc cells}

A typical electrode pass through the barn owl's inferior colliculus began with multiunit responses to dichotic stimuli, which gave way to responses from isolated cells selective for frequencies of about $2 \mathrm{kHz}$. More deeply, the best frequencies increased to a maximum of about $9 \mathrm{kHz}$, as expected from earlier descriptions of tonotopy in the ICc (Knudsen and Konishi, 1978; Wagner et al., 1987). The single units we encountered were generally excitable through either ear, with the contralateral ear usually providing a stronger input. The cells of the ICc could be distinguished from those of its lateral neighbor, the external inferior collicular nucleus, by their stronger response to tonal stimuli, their higher selectivity for frequency, and their relative insensitivity to interaural level difference. Although we isolated numerous cells in the course of an electrode pass, we studied only those ICc cells ( $n=59$ cells in 6 birds) that showed a significant selectivity for $\Delta \phi$ as judged by a statistical test of the vector strength (Rayleigh test with $p<0.01$; Durand and Greenwood, 1958). When binaural beat stimuli were presented, the neurons typically discharged spike bursts that were locked to the period of the binaural beat, and thus occurred at approximately the same $\Delta \phi$ within each binaural beat cycle. This beat-locked spiking continued throughout the course of the stimulation without any evidence of fatigue. The spiking of neurons in the ICc was not phase locked to the carrier frequency presented to either ear.

Our sample of cells had best frequencies between 2 and 8.3 $\mathrm{kHz}$, with 54 of 59 cells having best frequencies above $4 \mathrm{kHz}$. It is not surprising that such high-frequency cells would respond to $\Delta \phi$ in the barn owl, whose eighth nerve and cochlear nucleus phase lock up to $9 \mathrm{kHz}$ (Sullivan and Konishi, 1984). The use of these high frequencies excludes the involvement of the interaural canal, which connects the left and right middle ears of birds and can transmit sounds between the ears. In the barn owl, frequencies above $4 \mathrm{kHz}$ are severely attenuated within the canal and neuronal responses to these frequencies are not affected by crosstalk (Moiseff and Konishi, 1981b). Additionally, the five cells of our sample tested with frequencies at or below $4 \mathrm{kHz}$ showed no qualitative differences from cells tested with higher frequencies in response to motion or stationary stimuli. 

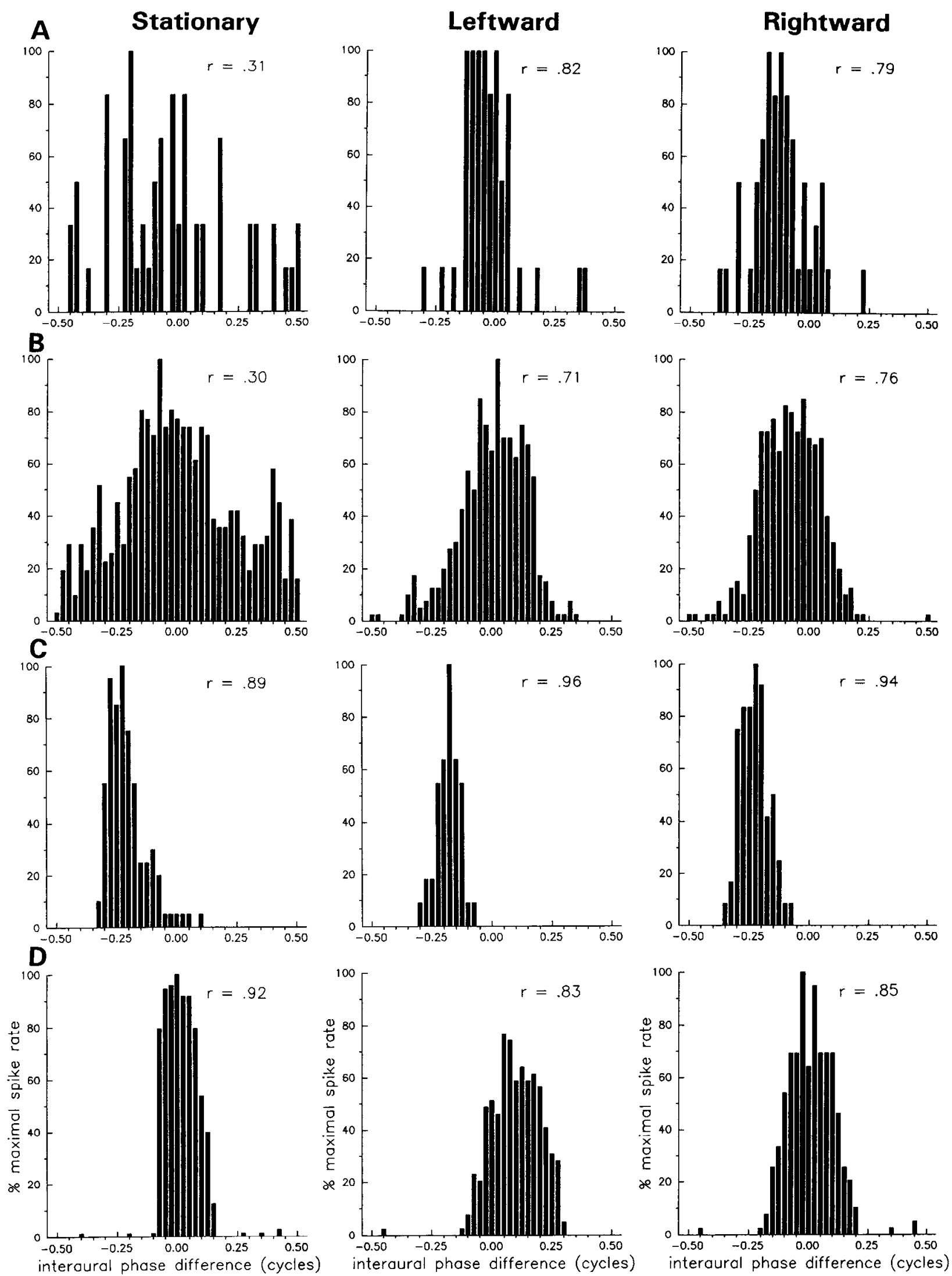

Figure 1. $\Delta \phi$ histograms of four ICc neurons $(A-D)$. For each neuron, a stationary $\Delta \phi$ histogram (left) and those obtained with leftward (center) and rightward (right) motion are shown. Negative $\Delta \phi$ values indicate that the tone in the left ear led. The vector strength, $r$, is shown at the top right of each histogram. The stationary histograms were normalized to the highest response. The motion histograms were normalized to the highest response obtained in either of the two directions. The carrier frequencies and the $\Delta F$ values are as follows: $A, 7692 \mathrm{~Hz}, 2 \mathrm{~Hz} ; B, 2000 \mathrm{~Hz}, 1 \mathrm{~Hz}$; $C, 2000 \mathrm{~Hz}, 1 \mathrm{~Hz} ; D, 5555 \mathrm{~Hz}, 1 \mathrm{~Hz}$. 


\section{Responses to tones in a quiet background}

Cells were generally more selective for $\Delta \phi$ when presented with moving stimuli than with stationary stimuli. Figure 1 shows histograms of spike rates as a function of $\Delta \phi$, obtained with stationary tones (left column) and with simulated leftward (center column) and rightward motion (right column) for four representative cells. Each stationary histogram is normalized to its own maximum, and the motion histograms are normalized to either the leftward or rightward maximum, whichever was higher. Comparison of absolute spike rates obtained with stationary and moving stimuli is not possible without making certain assumptions (see Discussion).

All of the histograms shown in Figure 1 are peaked, indicating that the neurons have a preferred $\Delta \phi$ regardless of whether or not the stimulus simulates motion. The shapes of the histograms are similar to those depicted in reports by others (Kuwada et al., 1979; Yin and Kuwada, 1983; Reale and Brugge, 1990; Spitzer and Semple, 1991; Moiseff and Haresign, 1992). For our sample as a whole, however, vector strengths obtained with leftward or rightward motion were greater than those obtained under stationary conditions ( $p<0.0001 ; N=52$ and $N=50$ for leftward and rightward pairs, respectively). The cells of Figure $1, A$ and $B$, demonstrate most clearly that with moving tones, the skirts of the histogram relative to the peak are lower in the motion condition than in the stationary condition. In fact, in Figure $1 B$, one can see that motion completely silences responses between +0.35 and +0.5 cycles, which were effective when the stimulus was stationary. $\Delta \phi$ histograms are thus narrower and vector strengths $(r)$ higher for the motion condition. Figure $1 C$ shows results from a third cell for which the vector strengths obtained with motion were higher than those obtained with stationary stimuli, but less dramatically so. Only four cells, one of which is depicted in Figure $1 D$, showed a higher vector strength for stationary conditions than for motion, and in neither cell was the difference as dramatic as in Figure $1, A$ and $B$.

The observation that motion will completely silence a cell at nonoptimal $\Delta \phi$ values suggests that inhibition is at least one mechanism by which motion enhances $\Delta \phi$ sensitivity beyond levels seen with stationary stimuli. The weakness of stationary vector strengths relative to motion vector strengths, however, might also be due to fatigue. A stationary stimulus left at preferred $\Delta \phi$ values for 1 sec might fatigue the cell, whereas at less effective $\Delta \phi$ values, where firing would be weaker, the cell would not fatigue. A cell's response would therefore be constrained only at the stronger stationary $\Delta \phi$ values. In such a case, vector strengths should diminish with time from the onset of a stimulus. To examine this possibility, we divided the response to the $1 \mathrm{sec}$ stationary tone at each bin into $250 \mathrm{msec}$ quartiles. Vector strengths obtained with moving tones were then compared to vector strengths for stationary stimuli computed from each quartile of the stationary responses. Once again, for our sample as a whole, vector strengths obtained with motion were higher than those obtaincd from any quartilc of the stationary responses $(p<0.05)$. Furthermore, no significant differences were found among vector strengths computed from different quartiles.

Our sample of cells showed little directional preference. Upon comparing the total spike numbers obtained with leftward and rightward motion, we found that only 5 of 45 cells discharged preferentially for one direction at a statistically significant level ( $p<0.05, \chi^{2}$ test). This proportion of directionally selective cells is comparable to that found in the ICc of the cat (Yin and Kuwada, 1983), but less than that found in the subdivisions of the owl's ICc and optic tectum combined (Wagner and Takahashi, 1990)

\section{Responses to tones in noise}

We asked how the cells' ability to detect a tonal stimulus is affected by noise. For these tests, moving and stationary tones were combined with interaurally uncorrelated noise, and the response of the neurons was measured over a range of $S / N$ values. Interaurally uncorrelated noise, which produces a variety of $\Delta \phi$ values randomly over time, can be thought of as the auditory analog of "snow" on a video screen. A neuron's preferred $\Delta \phi$ and frequency can arise at random, and the neuron's spontaneous discharge therefore tends to increase when interaurally uncorrelated noise is presented alone. A neuron's vector strength was used as an index of its ability to detect a tone. If a neuron is unable to detect the tone, its firing will be random with respect to the period of the tone, and its vector strength will be zero.

A neuron's $\Delta \phi$-specific response and therefore its ability to report the presence of the target within its receptive field was obscured by background noise. Figure 2 shows $\Delta \phi$ histograms at various $S: N$ values $(A-E)$ for one cell. At each ratio, the histogram on the left was obtained with a stationary stimulus while that on the right was obtained with rightward motion. Each histogram is normalized to the maximal spike rate in its column. The leftward response was indistinguishable from the rightward response and is not shown. At high $S / N$ values $(S / N$ $=10$; Fig. $2 A$ ), the neuron displayed the typical selectivity for $\Delta \phi$ already described, whether or not the stimulus simulated motion. When noise was added, there was a disproportionate increase in firing at formerly ineffective $\Delta \phi$ values, as well as an increased overall activity. In effect, noise broadened the histograms, obscuring the peak (Fig. $2 B-E$ ). The vector strengths correspondingly diminished.

Motion of the stimulus allowed the cell to maintain its $\Delta \phi$ spccificity. Figure 2, $C$ and $D$, shows that the peak of the motion histogram (right), relative to the skirts, remained distinct at $S$ / $N$ values that flattened the stationary histograms (left). The initial rate of recovery from the obscuring effect of the noise was higher for moving stimuli than for stationary stimuli, a point best seen in Figure $3 A$, which plots vector strength as a function of the $S / N$ for the cell of Figure 2. Figure $3, B$ and $C$, shows similar plots for two other cells. In each graph except Figure $3 D$, the slope of the curve at the lower $S / N$ values is higher for the motion conditions (triangles) than for the stationary condition (solid circles). In the cell of Figure $3 B$, the vector strength is higher for motion at the highest $S / N$ tested, whereas in the cells of Figure $3, A$ and $C$, the vector strengths for motion and stationary conditions are roughly equal at the high $S / N$. Figure $3 D$ shows one of two cases in which the responses to stationary and motion stimuli recovered in the same manner.

Figure $4, A$ and $B$, summarizes the differences in vector strengths obtained with moving and stationary stimuli for our sample. Each scatter plot graphs, as a function of $S / N$, the difference between motion and stationary vector strengths $\left(r_{\text {mot }}\right.$ and $r_{\text {stat, }}$ respectively) normalized to the sum of the two vector strengths:

$$
\text { Normalized difference }=\left(r_{\text {mot }}-r_{\text {stat }}\right) /\left(r_{\text {mot }}+r_{\text {stat }}\right) \text {. }
$$

Positive values indicate that moving stimuli had higher vector 
A $S / N=10.0$

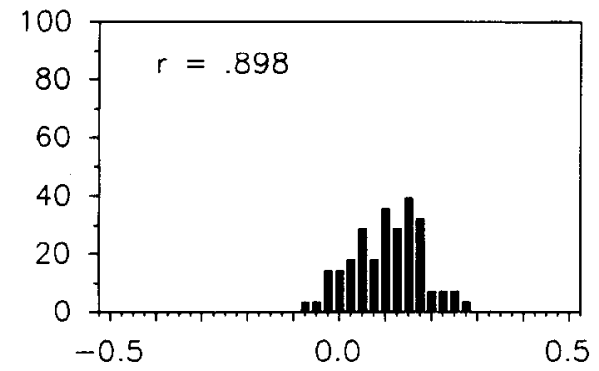

$B S / N=0.4$

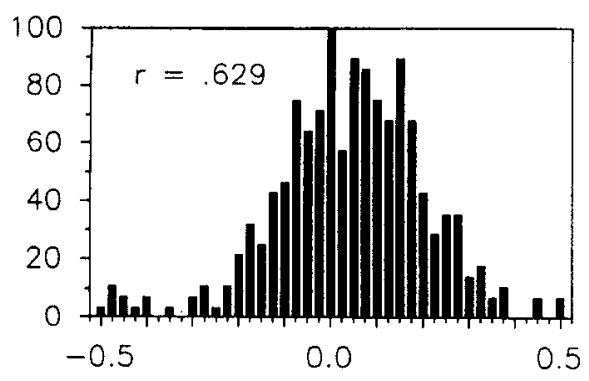

C $S / N=0.2$

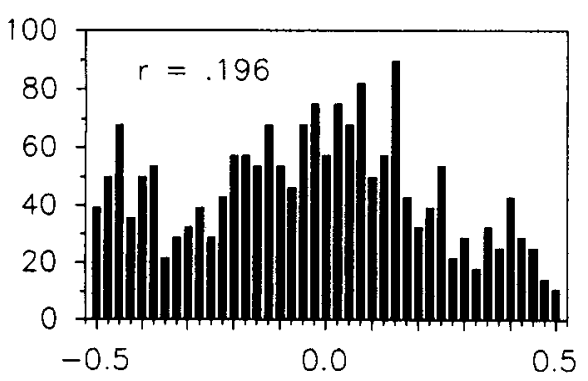

D $S / N=0.1$

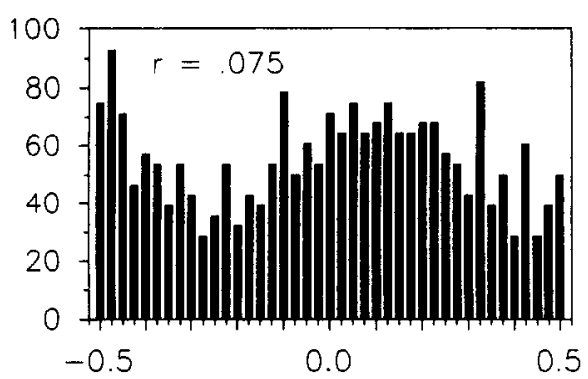

E $S / N=0.05$
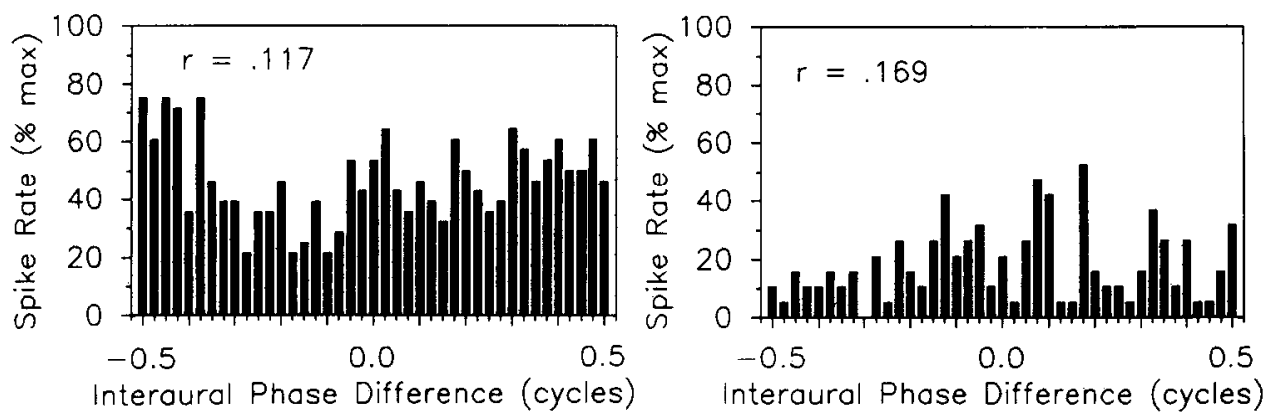
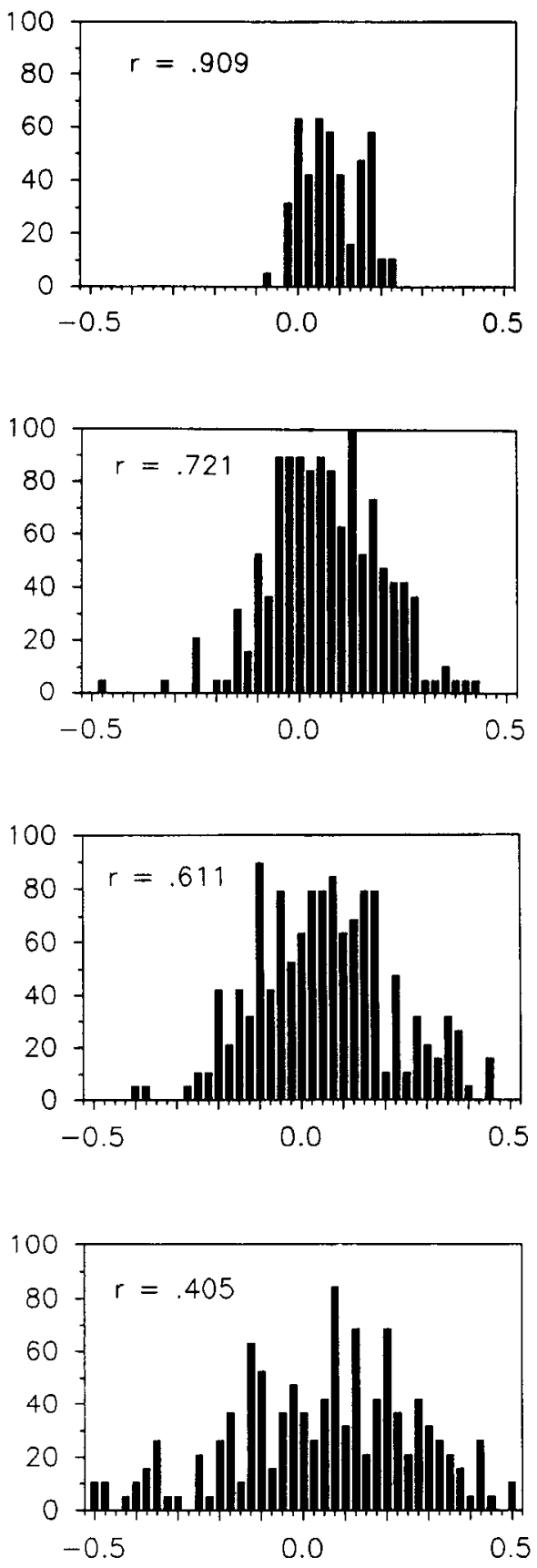

Figure 2. Response of an ICc neuron to tones in noise. $\Delta \phi$ histograms were plotted for stationary stimuli (left $)$ and rightward motion (right) at $S / N$ values of $10.0(A), 0.4(B), 0.2(C), 0.1(D)$, and $0.05(E)$. Binaural beats simulating leftward motion yielded histograms similar to those from rightward motion and are not shown. The spike rate is normalized to the maximal rate found in each column. The vector strength, $r$, is shown to the upper left of each histogram. The motion vector strength degrades less rapidly than the stationary vector strength as noise is added. Carrier frequency $=6667 \mathrm{~Hz} ; \Delta F=4 \mathrm{~Hz}$. 

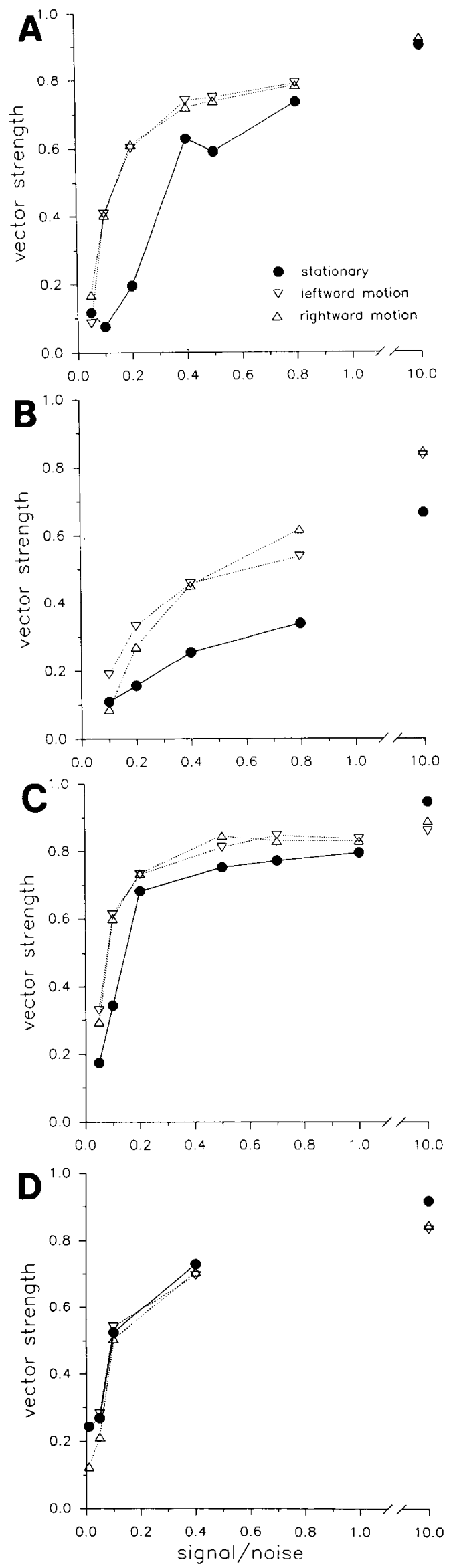

strengths and were therefore more detectable to the cell, and negative values indicate that stationary stimuli were more detectable. Figure $4 A$ is for leftward motion and Figure $4 B$ is for rightward motion. These scatter plots show that over a wide range of $S / N$ values, there is a strong tendency for moving stimuli to produce higher vector strengths than stationary stimuli in the presence of noise.

\section{Responses to amplitude modulation}

The results above suggest that ICc neurons are better able to signal the presence of stimuli in which a change of position over time is simulated. It is also possible, however, that the results are due to the periodic stimulation of a cell by a stimulus that repeatedly traverses its receptive field, and that a change in the position of the target is not nccessary. To test for this possibility, we amplitude modulated the stimulus synchronously in the two ears, thereby producing a time-varying stimulus without simulating changes in the stimulus's position. Such stimuli would be analogous to a stationary speaker periodically emitting tone bursts or to a flashing stationary light.

We compared the $\Delta \phi$ sensitivity of $21 \mathrm{ICc}$ cells to stationary AM tones, stationary unmodulated tones, and moving tones. The stimulus amplitude was modulated by a triangular envelope to a depth of $75 \%$ at a rate equal to the $\Delta F$ used for the binaural beats test. The results are shown for two cells in Figure 5, $A$ and $B$. Figure 5 plots vector strengths as a function of $S / N$, and as with the cells shown above, motion resists the effects of noise. In the same cells, moreover, vector strengths obtained with AM stimuli were no more effective in withstanding noise than were unmodulated stationary tones. This point is summarized for our sample of cells in Figurc $6 A$, which plots as a function of $S / N$ the normalized difference in vector strength between AM tones $\left(r_{\mathrm{am}}\right)$ and unmodulated tones $\left(r_{\mathrm{stat}}\right)$ :

Normalized difference for $\mathrm{AM}=\left(r_{\mathrm{am}}-r_{\text {stat }}\right) /\left(r_{\mathrm{am}}+r_{\text {stat }}\right)$.

The points in Figure $6 A$ at the lowest $S / N$ values tend to cluster about zero, indicating that an AM tone is not any easier for a cell to detect than an unmodulated tone. At the highest $S / N$ values tested, AM tones yield lower vector strengths than unmodulated stationary tones ( $p<0.05,19$ neurons). Similar scatter plots that compare AM tones to leftward and rightward motion at various $S / N$ values are shown in Figure $6, B$ and $C$. The equation for the normalized difference is identical to Equation 3, except that $r_{\text {mot }}$ is substituted for $r_{\text {stat }}$. In these scatter plots, the points generally fall below zero, indicating that moving stimuli yielded higher vector strengths than did AM stimuli. In a subset of cells, we tested modulation depths ranging from $12.5 \%$ to $100 \%$ and found results consistent with those described above for $75 \%$ modulation.

\section{Discussion}

There is a growing body of literature on the neurophysiology of acoustic motion sensitivity. Most of these studies have reported the existence of cells that discharge preferentially for a particular

Figure 3. Plots of vector strength as a function of $S / N$ for four ICc neurons $(A-D)$. The graph shown in $A$ was taken from the cell whose response was depicted in Figure 2. Graph $D$ is taken from the cell depicted in Figure $1 D$. Carrier frequencies and $\Delta F$ values are as follows: $A, 6667 \mathrm{~Hz}, 4 \mathrm{~Hz} ; B, 4762 \mathrm{~Hz}, 1 \mathrm{~Hz} ; C, 2564 \mathrm{~Hz}, 8 \mathrm{~Hz} ; D, 5555 \mathrm{~Hz}$, $1 \mathrm{~Hz}$. 

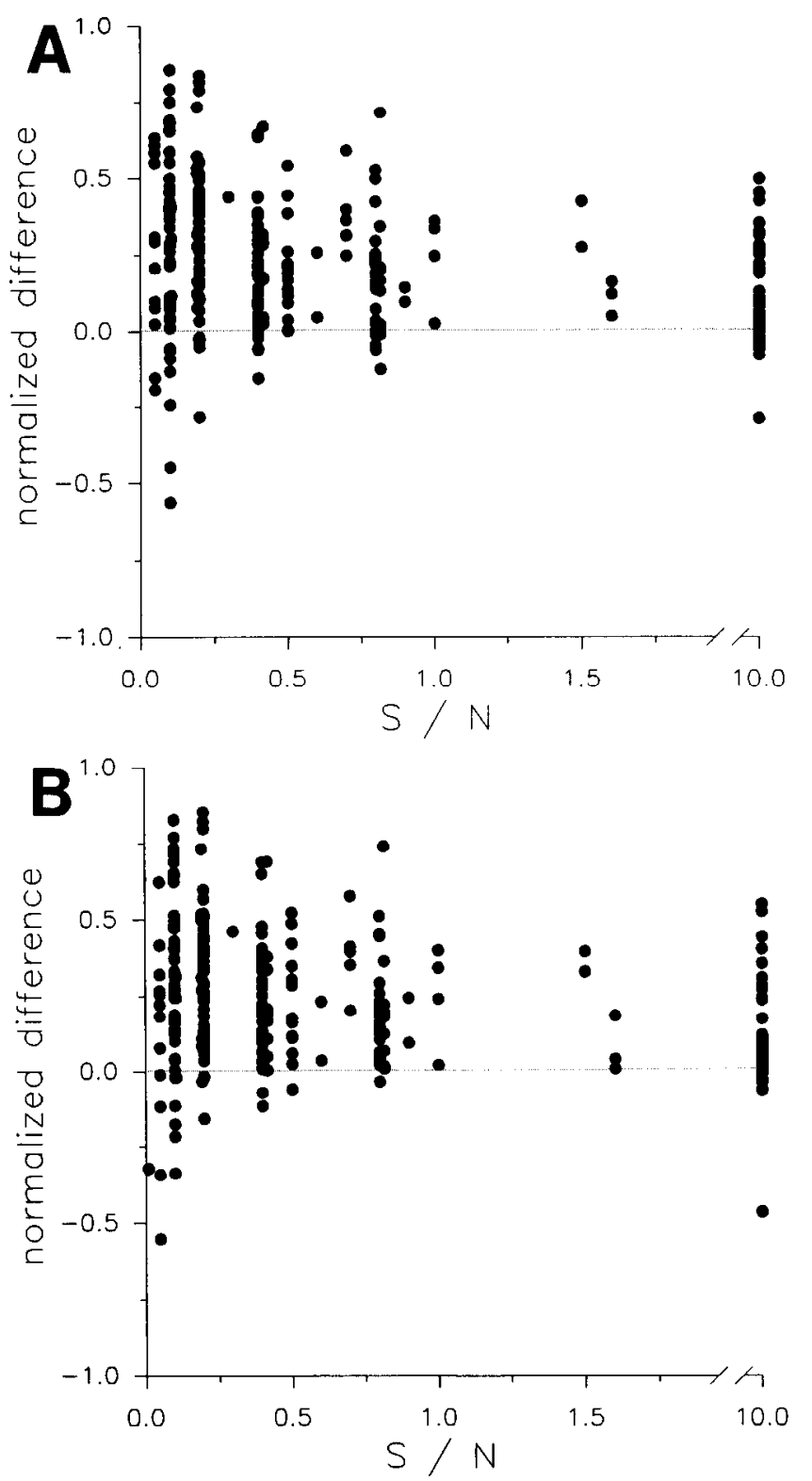

Figure 4. Scatter plots of normalized differences in vector strength as a function of $S / N$. The difference between the vector strengths obtained under motion conditions and stationary conditions normalized to the sum of the two (see Eq. 2) is plotted as a function of $S / N$. Positive values indicate that motion produced higher vector strengths. $A$, leftward motion; $B$, rightward motion.

direction of motion in a wide variety of brain areas including the ICc (e.g., Altman, 1968; Yin and Kuwada, 1983), superior colliculus (e.g., Jen et al., 1987; Rauschecker and Harris, 1989), cerebellum (Bechterev et al., 1975), and auditory cortex (e.g., Sovijarvi and Hyvarinen, 1974; Ahissar et al., 1992; Stumpf et al., 1992). The recent report by Spitzer and Semple (1991) and the present study address further issues: the effects of motion on auditory spatial resolution and figure-ground relationships.

The ICc is a nearly obligatory synaptic relay station for ascending auditory information. In this central role, it is likely to process signals that encode a wide variety of acoustic parameters, including the direction and speed of a sound source, for further extraction at higher levels. Our findings in the barn owl suggest that neurons of the ICc are capable of exploiting the

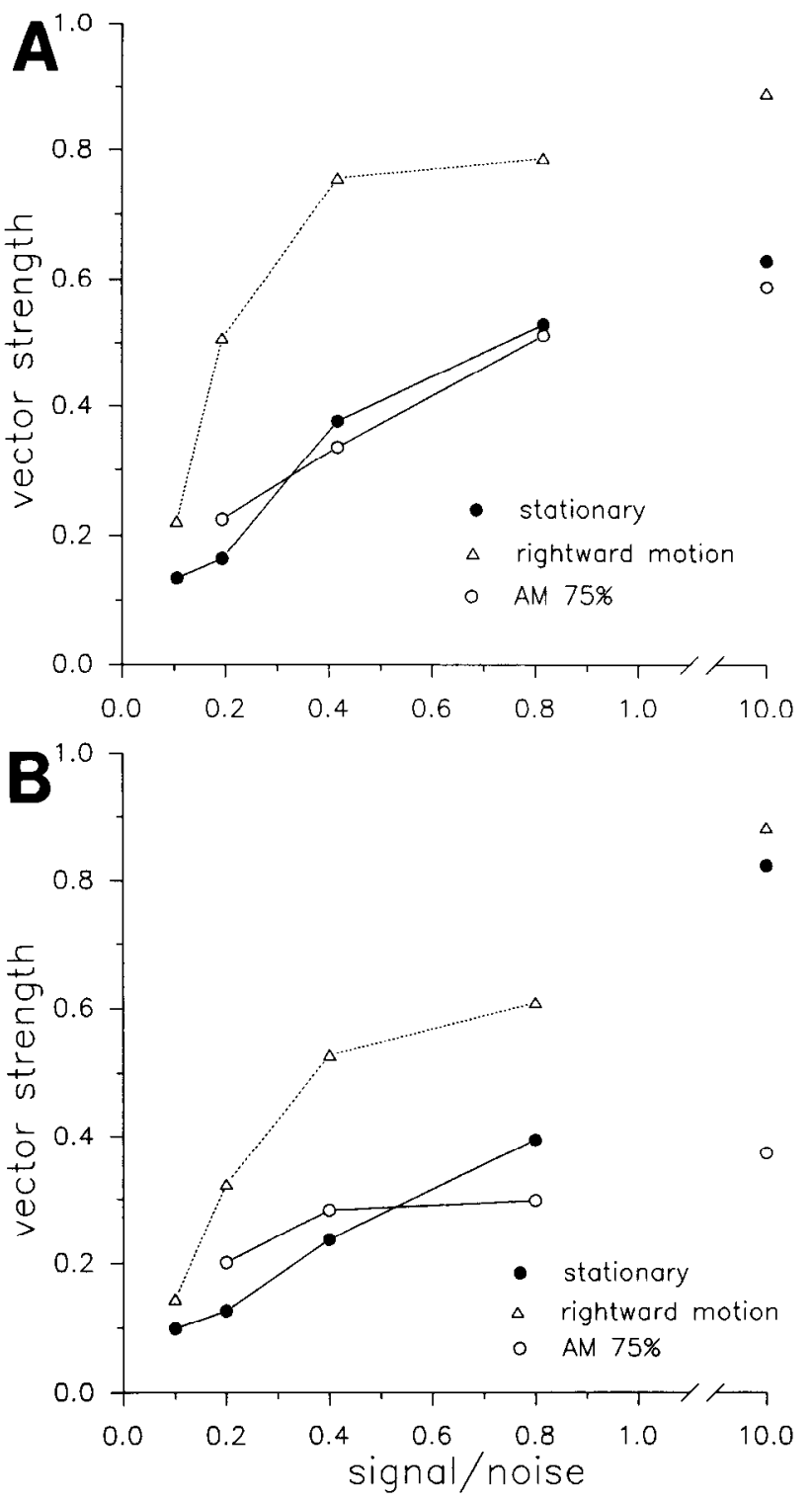

Figure 5. Comparisons AM tones, unmodulated tones, and binaural beats in noise. Vector strengths obtained with motion (triangles), unmodulated stationary tones (solid circles), and AM tones (open circles) were plotted as a function of $S / N$ for two ICc neurons $(A, B)$. Both plots show that vector strengths recover from the obscuring effects of noise most rapidly with motion, and that an AM stationary tone is no better than an unmodulated stationary tone. In fact, AMs tend to yield lower vector strengths at the high $S / N$ values as seen in $B$. Curves obtained with leftward motion were comparable to those obtained with rightward motion and are not shown. Modulation depth was $75 \%$. Carrier frequencics and $\Delta F$ valucs arc as follows: $A, 6667 \mathrm{~Hz}, 1 \mathrm{~Hz} ; B, 3000 \mathrm{~Hz}$, $1 \mathrm{~Hz}$.

motion of sound sources. Tones that mimic a stationary source can be obscured by noise, as judged by a neuron's vector strength, but if the $\Delta \phi$ changes continuously, thus mimicking motion, the neuron's $\Delta \phi$-specific response reemerges. The latter could not be observed when the stimulus mimicked a stationary, AM tonc source. The cells also show a higher selectivity for $\Delta \phi$ in the absence of noise if $\Delta \phi$ changes continuously over time than when a succession of fixed $\Delta \phi$ values is presented, suggesting that the place code for space in the ICc is of finer grain for moving sources.

As the phenomena described above were demonstrated with 

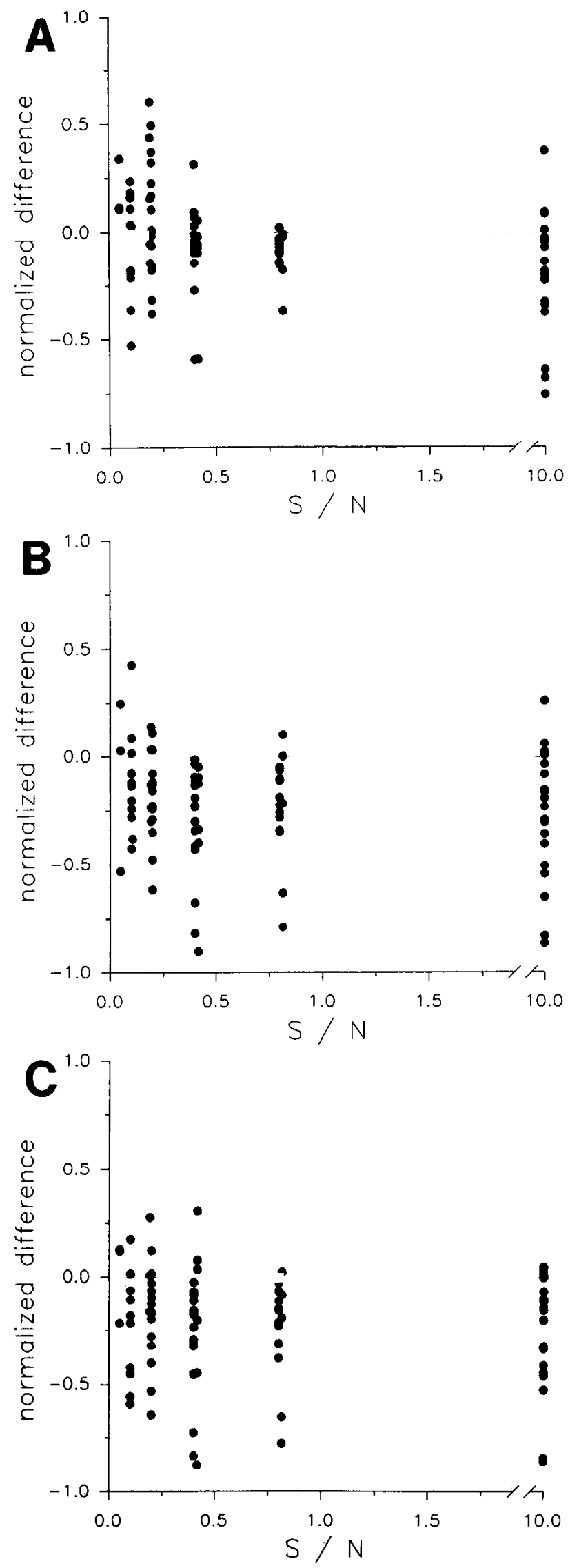

Figure 6. Comparison of AM tones with unmodulated stationary tones and with binaural beats for our sample. Positive values indicate that tonal stimuli, they can be subserved by neuronal interactions within a single isofrequency layer. Furthermore, since binaural beats, but not AMs, produce these phenomena, it is likely that the mechanism itself involves interactions between cells that represent adjacent $\Delta \phi$ values.

\section{Mechanisms and topographical maps}

In the ICc, $\Delta \phi$-selective neurons are organized tonotopically, and within each isofrequency lamina there is a topographic representation of $\Delta \phi$ (Wagner et al., 1987). Recent studies also suggest the existence of a GABA-mediated lateral inhibition within ICc that sharpens stationary $\Delta \phi$ tuning by suppressing discharge at suboptimal values of $\Delta \phi$ (Fujita and Konishi, 1991). Could the map in an isofrequency lamina of ICc suffice to explain our findings? A neuron's spiking can continue for a short time after a stimulus is turned off or leaves the cell's receptive field. Under stationary stimulus conditions, this persistence of firing is reflected only in the time domain. With motion, however, the persistence effectively widens the stimulus as it travels across the topographic map by leaving a wake of activity. The moving stimulus would first enter the inhibitory region of the cell's receptive field and, because of its wake, would recruit more inhibitory inputs than would a stationary stimulus, thereby exaggerating the inhibitory trough. As the moving stimulus enters the neuron's excitatory region, it will recruit more excitatory inputs than would a stationary stimulus, thereby exaggerating the excitatory peak. Perhaps by placing small phase modulations within selected portions of a cell's complex receptive field, we can produce similar effects. The deepened inhibitory troughs may be especially visible against a background discharge evoked by binaurally uncorrelated noise. In the end, a complete model must await considerable anatomical and physiological detail, but it seems reasonable to expect that the mechanisms that sharpen $\Delta \phi$ selectivity under stationary conditions can be enhanced to produce the results observed with motion.

\section{Comparisons with other neurophysiological studies}

A number of studies in the mammalian auditory system have demonstrated that $\Delta \phi$ histograms obtained with continuously changing $\Delta \phi$ are narrower than those obtained with stationary stimuli (Yin and Kuwada, 1983; Reale and Brugge, 1990; Spitzer and Semple, 1991). When normalized stationary and binauralbeat histograms are superimposed, it appears that for some mammalian cells the narrowing is due, at least in part, to inhibition at unfavorable $\Delta \phi$ values because some values that elicited spikes with stationary stimuli have zero discharge when the stimulus is binaural beats (Yin and Kuwada, 1983; Reale and Brugge, 1990).

A recent study by Spitzer and Semple (1991) demonstrates similar effects but also reports an elevated discharge at favorable $\Delta \phi$ values with motion. Thus, they argue for an enhancement of the response at the peak, in addition to a suppression of the

\footnotetext{
AMs yielded higher vector strengths. $A$, The normalized difference in vector strength between stationary AM tones and stationary unmodulated tones (Eq. 3) is plotted as a function of $S / N$. AM tones tend to yield similar vector strengths to that of unmodulated tones at low $S / N$ values. At high $S / N$, the vector strengths obtained with $A M$ are lower. $B$ and $C$, Normalized difference in vector strength between stationary AM tones and leftward $(B)$ and rightward $(C)$ motion. Vector strengths with AM tones are uniformly less than those obtained with motion.
} 
response at the troughs. To compare time-averaged spike rates as Spitzer and Semple (1991) did, one must assume that a cell's spike rate does not change significantly over the duration of the stationary stimulus. This was the case for the cells shown by $M$. W. Spitzer and M. N. Semple (personal communication). To evaluate this for our own data, we compared maximal spike rates elicited under stationary and motion conditions in two ways. In the first method, we made this assumption of a sustained rate. Thus, for the stationary condition, the spike rate was derived by counting spikes elicited during the entire $1 \mathrm{sec}$ tone at the optimal $\Delta \phi$. For motion, we counted the number of spikes elicited as the stimulus moved through the optimal $\Delta \phi$ bin and divided this quantity by the amount of time (in seconds) spent in the bin. When these spike rates are compared in a scatter plot, virtually every cell's data point falls above the dotted line that represents equal responses to moving and stationary stimuli (Fig. 7 $A$, rightward motion; leftward motion gave similar results and is not shown). From this figure, it seems clear that motion elicits higher maximal rates. Alternatively, we asked whether each cell could achieve similar maximal spike rates for motion and stationary conditions whether or not these rates were maintained throughout the period of stimulation. Ideally, this analysis would compare maximal instantaneous spike rates attained under the two conditions. Only a small percentage of our cells, however, fired at rates high enough to allow meaningful estimates of instantaneous spike rates. Instead, we subdivided the optimal $\Delta \phi$ bin for stationary conditions into intervals of 50 msec, which is less time than the moving stimulus spends within the receptive field of most cells. We then calculated a spike rate from the interval with the greatest number of spikes. This analysis is plotted in Figure $7 B$ for rightward motion. These points tend to disperse evenly above and below the unity slope line, indicating that the motion and stationary discharge rates are not very different. In a few cells with high spike rates, however, motion elicited more spikes even when compared with the latter, more conservative method (Fig. $7 B$ ), and several other cells showed no response at all to stationary tones but did fire for binaural beats (Fig. 7 $A$ ). The issue of motion and stationary spike rates therefore depends on the time over which averages are taken whether the averaging is done by the experimenter or a postsynaptic neuron.

\section{Comparison with psychoacoustical masking studies}

Psychoacoustical studies have shown that simulated motion does not enhance the detection of noise-masked signals for man. Wilcott and Gales (1954) masked a bandpassed noise (75-850 $\mathrm{Hz}$ ) with binaurally uncorrelated noise and stepped the interaural delay such as to simulate targets with angular speeds of $6.3^{\circ}, 12.6^{\circ}$, and $37.3^{\circ}$ of azimuth per second ${ }^{1}$. Detection thresholds thus obtained were compared to those obtained with a similarly masked, stationary signal at a central or eccentric position. These authors found no difference in detectability between moving and stationary targets. More recently, Grantham and Luethke (1988) made similar observations using tones with continuously varying $\Delta \phi$, masked by binaurally uncorrelated noise. Their studies took care to confine the perceived motion to within the physiological limits of ITD. Compared to the study of Wilcott and Gales (1954), the angular speeds simulated were

'Computed using Equation 1 and assuming a maximal ITD of $650 \mu \mathrm{sec}$ for humans, which results in $k=7.2 \mu \mathrm{sec}$ of ITD per degree of azimuth (Blauert, 1983).
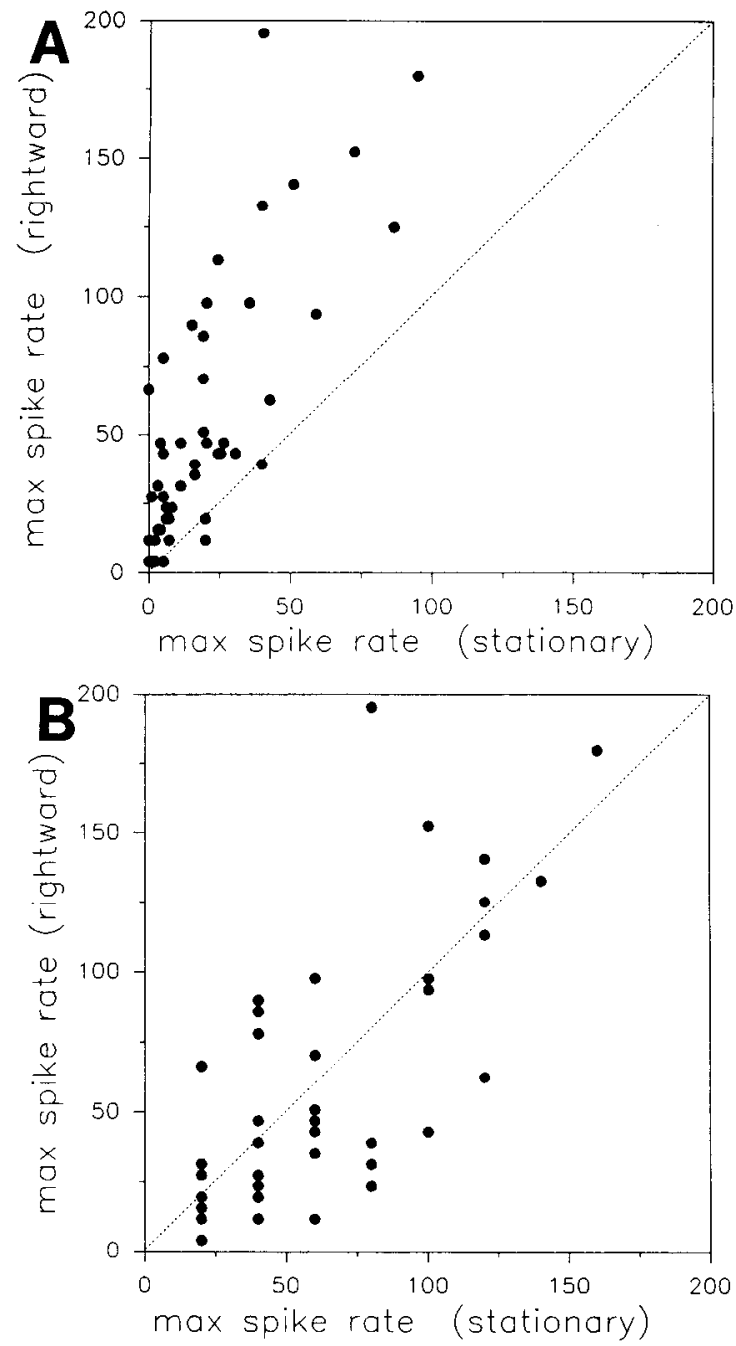

Figure 7. Scatter plots of maximal firing rates obtained with binaural beats graphed as a function of stationary firing rates. The dotted lines have a slope of 1 . The motion spike rate is the quotient of the number of spikes in the optimal $\Delta \phi$ bin divided by the amount of time (in seconds) spent in that bin by the moving stimulus. In $A$, the stationary spike rate is the number of spikes obtained with the stimulus fixed at the optimal $\Delta \phi$ for $1 \mathrm{sec}$. This comparison clearly shows higher spike rates for moving stimuli. A few cells do not discharge at all for stationary stimuli. In $B$, stationary spike rates were calculated from the highest spike rate observed after subdividing the response to the $1 \mathrm{sec}$ stationary tone into $50 \mathrm{msec}$ intervals. This comparison shows that motion and stationary stimuli tend to elicit comparable firing rates. Cells that did not discharge for stationary tones were excluded from $B$.

somewhat higher $\left(173^{\circ}, 347^{\circ}, 1730^{\circ}\right.$, and $3472^{\circ}$ of azimuth per second $)^{1}$ due to low carrier frequencies and/or high $\Delta F$ values. The $\Delta \phi$ of the stationary signals used for comparison were fixed at $0, \pi / 2$, or $\pi$.

When the masker is instead binaurally correlated, the results can be explained from what is known regarding the binaural masking of stationary targets (Robinson, 1971; Grantham and Luethke, 1988). With this stimulus configuration, signal detectability is poorest when the signal and masker have similar $\Delta \phi$ values and is better if the $\Delta \phi$ values of the two differ (Jeffress et al., 1952). This is not surprising considering that the binaurally correlated masker would generate a single, well-localized, auditory image at the midline and that the difference in the $\Delta \phi$ values of the target and noise masker would dictate the degree 
of separation between the two auditory images. With tones of continuously varying $\Delta \phi$, the thresholds are somewhere between the lowest and highest detcction thresholds obtained with stationary tones, suggesting that the subjects compute an average from the perceived instantaneous $\Delta \phi$ values (Grantham and Luethke, 1988). Thus, in man, acoustic motion does not seem to facilitate the detection of masked targets despite the range of speeds, signal types, and maskers tested.

By contrast, there is evidence to suggest that motion facilitates target perception in echolocating bats. Jen and Kamada (1982) demonstrated that two species of echolocating bats, Pteronotus parnellii and Eptesicus fuscus, are better able to avoid moving obstacles than stationary ones during flight. McCarty and Jen (1983) also showed behaviorally that a bat's ability to discriminate the echo reflected off the target from those reflected off background objects degrades less rapidly with the bat-target distance if the target moves. Similarly, Simmons (1981) showed that $E$. fuscus's detection threshold for an echolocation signal in noise is lower if the arrival timc of returning echoes is jittered, simulating a target whose range fluctuates with time. These behavioral studies in bats and our neurophysiological findings in the barn owl contrast with the negative findings in man. This difference may reflect the more central role played by spatial hearing in the behavior of bats and owls.

\section{References}

Ahissar M, Ahissar E, Bergman H, Vaadia E (1992) Encoding of sound-source location and movement: activity of single neurons and interactions between adjacent neurons in the monkey auditory cortex. J Neurophysiol 67:203-215.

Altman JA (1968) Are there neurons detecting direction of sound source motion? Exp Neurol 22:13-25.

Anstis S (1970) Phi movement as a subtraction process. Vision Res 10:1411-1430.

Bechterev NN, Syka J, Altman JA (1975) Responses of cerebellar units to stimuli simulating sound source movement and visual moving stimuli. Experientia 31:819-821.

Blauert J (1983) Spatial hearing. Cambridge, MA: MIT Press.

Boring EG (1948) Sensation and perception in the history of experimental psychology. New York: Appleton-Century.

Braddick OJ (1974) A short-range process in apparent motion. Vision Res 25:839-847.

Durand D, Greenwood JA (1958) Modification of the Rayleigh test for uniformity in analysis of two-dimensional orientation data. J Geol 66:229-238.

Fujita I, Konishi M (1991) The role of GABAergic inhibition in processing of interaural time difference in the owl's auditory system. $\mathbf{J}$ Neurosci 11:722-739.

Goldberg JM, PB Brown (1969) Response of binaural neurons of dog superior olivary complex to dichotic tonal stimuli: some physiological mechanisms of sound localization. J Neurophysiol 32:616-636.

Grantham DW (1986) Detection and discrimination of simulated motion of auditory targets in the horizontal plane. J Acoust Soc Am 79: 1939-1949.

Grantham DW, Luethke LE (1988) Detectability of tonal signals with changing interaural phase differences in noise. J Acoust Soc Am 83: 1117-1123.

Jeffress LA, Blodgett HC, Deatherage BH (1952) The masking of tones by white noise as a function of the interaural phases of both components. I. 500 cycles. J Acoust Soc Am 24:523-527.

Jen PHS, Kamada T (1982) Analysis of orientation signals emitted by the CF-FM bat, Pteronotus p. parnellii and the FM bat, Eptesicus fuscus during avoidance of moving and stationary obstacles. J Comp Physiol 148:389-398.

Jen PHS, Zhang WP, Sun XD, Zhang SQ (1987) Auditory inputs and response of superior collicular neurons of the big brown bat, Eptesicus fuscus, to stationary and moving acoustic stimuli. Soc Neurosci Abstr 13:1469.
Knudsen EI, Konishi M (1978) Space and frequency are represented separately in the auditory midbrain of the owl. J Neurophysiol 41 : 870-884.

Knudsen EI, Konishi M (1979) Mechanisms of sound localization in the barn owl (Tyto alba). J Comp Physiol 133:13-21.

Kulikowski JJ, Tolhurst DJ (1973) Psychophysical evidence for sustained and transient detectors in human vision. J Physiol (Lond) 232: 149-162.

Kuwada S, Yin TCT (1983) Binaural interaction in low-frequency neurons in inferior colliculus of the cat. I. Effects of long interaural delays, intensity, and repetition rate on interaural delay function. $J$ Ncurophysiol 50:981-999.

Kuwada S, Yin TCT, Wickesberg RE (1979) Response of cat inferior colliculus neurons to binaural beat stimuli: possible mechanisms for sound localization. Science 206:586-588.

McCarty JK, Jen PHS (1983) Bats reject clutter interference for moving targets more successfully than for stationary ones. J Comp Physiol $152: 447-454$.

Moiseff A (1989) Binaural disparity cues available to the barn owl for sound localization. J Comp Physiol 164:629-636.

Moiseff A, Haresign T (1992) Response of auditory units in the barn owl's inferior colliculus to continuously varying interaural phase differences. J. Neurophysiol 67:1428-1436.

Moiseff A, Konishi M (1981a) Neuronal and behavioral sensitivity to binaural time differences in the owl. J Neurosci 1:40-48.

Moiseff A, Konishi M (1981b) The owl's interaural pathway is not involved in sound localization. J Comp Physiol 144:299-304.

Payne RS (1971) Acoustic location of prey by barn owls (Tyto alba). J Exp Biol 54:535-573.

Rauschecker JP, Harris LR (1989) Auditory and visual neurons in the cat's superior colliculus selective for the direction of apparent motion stimuli. Brain Res 490:56-63.

Reale RA, Brugge JF (1990) Auditory cortical neurons are sensitive to static and continuously changing interaural phase cues. J Neurophysiol 64:1247-1260.

Robinson DE (1971) The effect of interaural signal-frequency disparity on signal detectability. J Acoust Soc Am 50:568-571.

Simmons JA (1981) Detection of range motion in bat sonar. J Acoust Soc Am 69[Suppl 1]:S112.

Sokal RR, Rohlf FJ (1981) Biometry. The principles and practice of statistics in biological research, 2d ed. New York: Freeman.

Sovijarvi AR, Hyvarinen J (1974) Auditory cortical neurons in the cat sensitive to correlates of sound source movement. Brain Res 73: $455-471$.

Spitzer MW, Semple MN (1991) Interaural phase coding in auditory midbrain: influence of dynamic stimulus features. Science $254: 721-$ 724.

Stumpf E, Toronchuk JM, Cynader MS (1992) Neurons in cat primary auditory cortex sensitive to correlates of auditory motion in three dimensional space. Exp Brain Res 88:158-168.

Sullivan WE, Konishi M (1984) Segregation of stimulus phase and intensity coding in the cochlear nucleus of the barn owl. J Neurosci 4:1787-1799.

Takahashi TT, Konishi M (1988a) Projections of the cochlear nuclei and nucleus laminaris to the inferior colliculus of the barn owl. J Comp Neurol 274:190-211.

Takahashi TT, Konishi M (1988b) Projections of nucleus angularis and nucleus laminaris to the lateral lemniscal nuclear complex of the barn owl. J Comp Neurol 274:212-238.

Takahashi TT, Konishi M, Carr CE, Brecha N (1987) Calcium binding protein-like immunoreactivity labels the terminal field of nucleus laminaris of the barn owl. J Neurosci 7:1843-1856.

Wagner H, Takahashi TT (1990) Neurons in the midbrain of the barn owl are sensitive to the direction of apparent acoustic motion. Naturwissenschaften 77:439-442.

Wagner H, Takahashi T, Konishi M (1987) Representation of interaural time difference in the central nucleus of the barn owl's inferior colliculus. J Ncurosci 7:3105-3116.

Wilcott RC, Gales RS (1954) Comparison of the masked thresholds of a simulated moving and stationary auditory signal. J Exp Psychol 47:451-456.

Yin TCT, Kuwada S (1983) Binaural interaction in low-frequency neurons in inferior colliculus of the cat. II. Effects of changing rate and direction of interaural phase. $\mathrm{J}$ Neurophysiol 50:1000-1018. 